Published in IET Science, Measurement and Technology

Received on 9th July 2007

Revised on 4th November 2007

doi: 10.1049/iet-smt:20070056

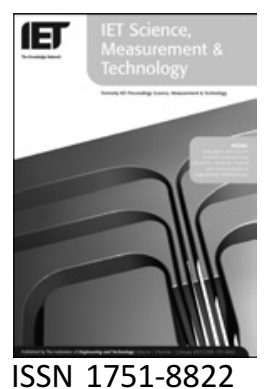

\title{
Electromagnetic compatibility analysis of
} unstructured mains networks for high-speed data transmission: Part 2

$\begin{array}{lll}\text { S. Battermann } & \text { H. Garbe } & \text { F. Silva } \\ & \text { M. Pous } & \end{array}$

V. Beauvois ${ }^{3}$ K. Vantomme J. Catrysse $^{4} \quad$ J. Newbury ${ }^{5}$

V. Degardin ${ }^{6}$ M. Liénard ${ }^{6} \quad$ P. Degauque $\quad$ I.D. Flintoft ${ }^{7}$ $\begin{array}{lll}\text { A.D. Papatsoris } & \text { D.W. Welsh } & \text { A.C. Marvin }\end{array}$

${ }^{1}$ The Institute of the Basics of Electrical Engineering and Measurement Science, Leibniz University of Hannover, Appelstraße 9A, Hannover 30167, Germany

${ }^{2}$ The Universitat Politècnica de Catalunya, Campus Nord C4 Jordi Girona 1-3, Barcelona 08034, Spain

${ }^{3}$ The Department of Electrical Engineering and Computer Science, University of Liège, Insitut Montefiore B28, Liège 4000, Belgium

${ }^{4}$ Katholieke Hogeschool Brugge-Oostende, Zeedijk 101, Oostende B-8400, Belgium

${ }^{5}$ The Open University in the North West, 351 Altincham Road, Sharston, Manchester M22 4 UN, UK

${ }^{6}$ The Institut d'Electronique, de Microélectronique et de Nanotechnologies (IEMN), University of Lille, Bat. P3, Villeneuve d Ascq cedex 59655, France

${ }^{7}$ Department of Electronics, University of York, Heslington, York YO10 5DD, UK

E-mail: battermann@geml.uni-hannover.de

Abstract: Different approaches are described for the characterisation of the low voltage mains network that is used for high-speed data transmission broadband over power line (BPL). Part 1 investigated the disturbance scenario of these BPL devices and importance will be given to develop a new measurement procedure in Comité Internationale Spécial des Perturbations Radioelectrotechnique (CISPR) to estimate the highfrequency characteristics of AC mains networks. The improvements of the measurement method that can also be applied to other wire-based telecommunication systems will also be presented. Part 2 shows the definitions and comparisons of different symmetry factors. The transfer of the results on the compliance test for BPL devices will also be depicted.

\section{Introduction}

In Part 1 of this paper, a new measurement set-up to determine the symmetrical and asymmetrical attenuations of the mains network was presented including measurement results. Part 2 shows different factors to describe the mains high-frequency (HF) symmetry in detail. After an examination of the coupling path of symmetrical and asymmetrical signals from one socket to another socket of a home power mains network, the mains symmetry factor $\left(M_{\mathrm{S}, \mathrm{F}}\right)$ and the mains conversion factor will be presented. A comparison of the mains symmetry factor based on currents and alternatively based on magnetic field strength is shown. The transfer of the obtained symmetry factor to a telecommunication impedance stabilisation networks (T-ISN) used for compliance tests is described. 


\section{Calculated factors to determine the mains symmetry}

\subsection{Description of the HF symmetry of a mains network}

The HF symmetry of a mains network is the relation of the level of a differential-mode signal, $G_{\text {sym,in }}$, fed into the network at position A to the level of a commonmode signal, $G_{\text {asym,in }}$, also fed at position A, for which both feeding modes cause the same common-mode signal, $G_{\text {asym,out }}$, at position B (Fig. 1b). In the case of current measurements, $G_{\text {asym,out }}$ denotes the commonmode current at the receiver, which is the disturbance quantity [1]. Because of the mode conversion along the wires of the mains network, even a perfectly balanced differential-mode signal fed at position A will be converted to a common-mode signal at position B. For this reason, two different coupling paths could be defined: the first one when a common-mode signal $\left(G_{\text {asym,in }}\right)$ is applied at socket A and the second one when the signal is applied in differential mode $\left(G_{\text {sym,in }}\right)$. The conversion to common-mode output signal may appear mainly (i) at the end of the mains wire at position $\mathrm{B}$ (Fig. 1b, path 1), (ii) at the beginning at position A (Fig. 1b, path 2) or (iii) through all the wire lengths as a combination of paths 1 and 2. This effect will be explained in detail in Section 2.3.

\subsection{Attenuation of a symmetrical or asymmetrical signal}

The attenuation of a symmetrical (or differential mode) signal between the feeding (at position A) and the load socket (at position B) is $A_{\text {sym }}$ (in dB) (Fig. 1a)

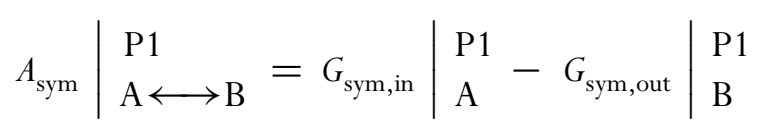

The attenuation of an asymmetrical (or common mode) signal $A_{\text {asym }}$ between the feeding (at position A) and the load socket (at position B) (in $\mathrm{dB}$ ) is calculated as
(Fig. 1a)

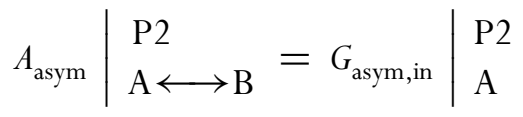

$$
\begin{aligned}
& \begin{array}{l|l}
-G_{\text {sym }, \text { out }} & \begin{array}{l}
\text { P2 } \\
\text { B }
\end{array}
\end{array}
\end{aligned}
$$

For practical measurements, it is essential to have welldefined differential-and common-mode impedances for all measurements. The impedance conditions of the feeding and the load unit have to be kept constant for all measurements.

In this paper, the current has been used for the parameter $G$, although it is also possible to use the voltage, power or field strength if the quantity is representative of the disturbance. The symmetrical attenuation for the installation that has been described in Part 1 of this paper is shown in Fig. 2a. In the lowfrequency range, two groups exist: sockets 6,8 and 9 and sockets 1, 3 and 7 . This correlates with the installation. Sockets 1,3 and 7 are connected in parallel with the devices with low differential-mode impedance (e.g. line filters), which results in a high attenuation. The attenuation of the asymmetrical signal is depicted in Fig. $2 b$ and is generally lower than the attenuation of the symmetrical signal. Again two different cluster of sockets exist.

\subsection{Calculation of the mains symmetry factor $M_{S, F}$ or the HF symmetry of the network}

In reality, the mode conversion takes place along the length of the complete mains network. If the lengthdependent line parameters of the mains network were available, then it would be possible to calculate the HF symmetry accurately at all positions of the network. Different theoretical approaches show the necessary background $[2,3]$. Generally, the HF symmetry of an $\mathrm{AC}$ mains network is a parameter that does not depend on the cable length actually considered. It is a primary parameter for the HF properties of mains networks and in-house installations. However,

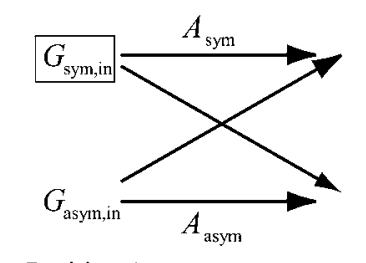

Position A

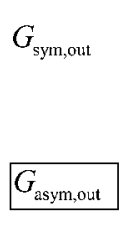

Position B

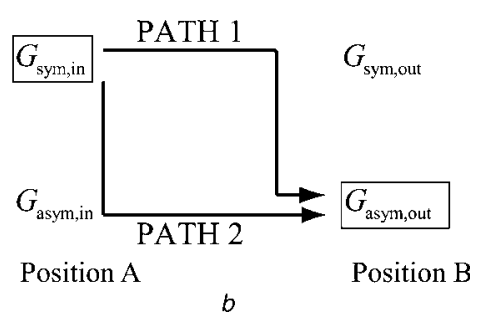

Figure 1 Schematic matrix of the measured signals and the determined attenuations 

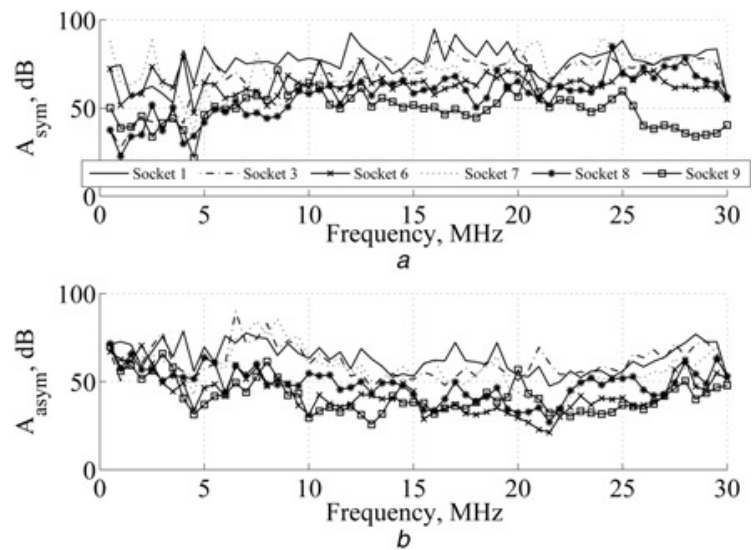

Figure 2 Attenuation of the signal

a Symmetrical feeding

$b$ Asymmetrical feeding

for real installations, it is impractical to measure lengthdependent line parameters. Therefore it is necessary to determine a kind of integral mean value for the HF symmetry of the part of the network concerned. As a result of the model in Fig. 1b, the homogenous spread mode conversion can be reduced to three different cases only. The decision, which case applies, depends on the relation between $A_{\mathrm{sym}}$ and $A_{\text {asym }}$, which shall also be determined during the measurements in real networks

$$
\begin{array}{ll}
\text { Case 1 } & A_{\text {sym }}<A_{\text {asym }} \\
\text { Case 2 } & A_{\text {sym }}>A_{\text {asym }} \\
\text { Case 3 } & A_{\text {sym }}=A_{\text {asym }}
\end{array}
$$

Case 1: The symmetrical overall attenuation is significantly smaller than the asymmetrical overall attenuation. For this case, the symmetrical signal has a relatively low attenuation and a larger part of the symmetrical signal exists at the end of the line. This symmetrical signal can be converted to an asymmetrical signal at the end of the line and causes a significant part of the asymmetrical signal at the output. The asymmetrical attenuation is relatively high and the converted signals at the beginning of the cable have a high attenuation and negligible amplitude at the end of the cable. Signal path 1 in Fig. $1 b$ dominates. The relation between $G_{\text {sym,in }}$ and $G_{\text {asym,in }}$ has to be corrected by the symmetrical attenuation as shown in (4)

$$
\begin{aligned}
& M_{\mathrm{S}, \mathrm{F}}=\left.G_{\text {sym }, \text { in }}\right|_{\mathrm{A}}-\left.G_{\text {asym,out }}\right|_{\mathrm{B}}-A_{\text {sym }} \mid \begin{array}{l}
\mathrm{P} 1 \\
\mathrm{~A} \longleftrightarrow \mathrm{B}
\end{array} \\
& \text { with } \mid \begin{array}{l}
\text { A: } Z_{\mathrm{S}, \text { asym } 1}=Z_{\mathrm{S}, \text { asym } 2} \\
\text { B: } Z_{\mathrm{RX}, \text { asym } 1}=Z_{\mathrm{RX}, \text { asym } 2}
\end{array}
\end{aligned}
$$

Case 2: The symmetrical overall attenuation is significantly larger than the asymmetrical overall attenuation. Only at the beginning of the cable significant parts of the symmetrical signal can be converted into an asymmetrical signal. Path 2 in Fig. $1 b$ dominates and the asymmetrical signal at the end of the cable is mainly influenced by the asymmetrical attenuation. Therefore the relation between $G_{\text {sym,in }}$ and $G_{\text {asym,in }}$ has to be corrected by the asymmetrical attenuation $A_{\text {asym }}$

$$
\begin{aligned}
& M_{\mathrm{S}, \mathrm{F}}=G_{\mathrm{sym}, \mathrm{in}}\left|\mathrm{A}-G_{\text {asym,out }}\right|_{\mathrm{B}}-A_{\text {asym }} \mid \begin{array}{l}
\mathrm{P} 2 \\
\mathrm{~A} \longleftrightarrow \mathrm{B}
\end{array} \\
& \text { with } \mid \begin{array}{l}
\text { A: } Z_{\mathrm{S}, \text { asym } 1}=Z_{\mathrm{S}, \text { asym } 2} \\
\text { B: } Z_{\mathrm{RX}, \text { asym } 1}=Z_{\mathrm{RX}, \text { asym2 }}
\end{array}
\end{aligned}
$$

Case 3: The symmetrical overall attenuation is equivalent to the asymmetrical overall attenuation. For this case both paths will have the same attenuation and either one of the two equations may be used.

The mean value of $M_{\mathrm{S}, \mathrm{F}}$ (in $\mathrm{dB}$ ) is calculated from (6) using measurements at $n$ different sockets with the corresponding symmetrical or asymmetrical attenuation. For a statistically reliable result, it is necessary to measure in several typical house installations too

$$
\begin{aligned}
\bar{M}_{\mathrm{S}, \mathrm{F}} & =\frac{1}{n} \cdot \sum_{i=1}^{n} M_{\mathrm{S}, \mathrm{F}} \\
& =\frac{1}{n} \cdot \sum_{i=1}^{n}\left(\left.G_{\text {sym }, \text { in }, i}\right|_{\mathrm{A}}-\left.G_{\text {asym }, \text { out }, i}\right|_{\mathrm{B}}-\left.A_{i}\right|_{\mathrm{A} \longleftrightarrow \mathrm{B}}\right)
\end{aligned}
$$

The mains symmetry factor or the HF symmetry of a network obtained from the measurements is shown in Fig. 3. For each socket and each frequency step the $M_{\mathrm{S}, \mathrm{F}}$ has been calculated with respect to Cases $1-3$ based on the relation between $A_{\text {sym }}$ and $A_{\text {asym }}$. The $M_{\mathrm{S}, \mathrm{F}}$ starts at $\sim 40 \mathrm{~dB}$ and slopes to about $0 \mathrm{~dB}$ in the frequency range $\sim 4 \mathrm{MHz}$. This is the frequency range where $A_{\text {asym }}$ starts to become smaller than $A_{\text {sym }}$. Then $M_{\mathrm{S}, \mathrm{F}}$ goes up to $20 \mathrm{~dB}$ and starts to slope again above $25 \mathrm{MHz}$. This means that the low-voltage mains network has a good symmetry in the lower frequency range that drops down at around $4 \mathrm{MHz}$, goes up again and drops down again above $25 \mathrm{MHz}$.

Fig. 4 shows that the mean $M_{\mathrm{S}, \mathrm{F}}$ value of the different sockets has nearly a normal distribution with a mean value of $15 \mathrm{~dB}$. For a worst-case estimate, Fig. 5 shows the probability plots for socket 9. The measurement at socket 9 is the only measurement on 


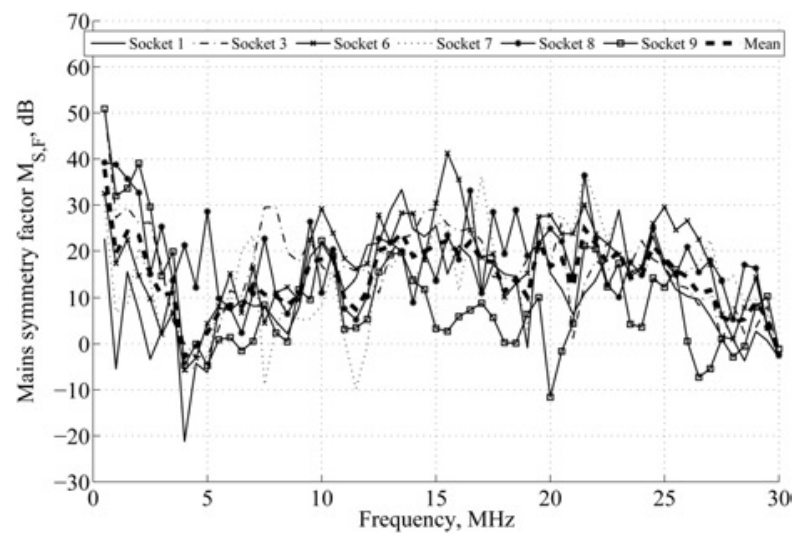

Figure 3 Resulting mains symmetry factor $M_{S, F}$ for six different sockets

Broad dashed line is the mean value for each frequency

the same phase with the source. It has a mean value of only $7.5 \mathrm{~dB}$ at $50 \%$ probability, which is the lowest value of all sockets. As a result, the worst-case mains symmetry factor for this data set is about $7.5 \mathrm{~dB}$. Therefore it is quite important to distinguish measurements between different phases and measurements on a single phase. The magnitude and typical frequency behaviour of the obtained $M_{\mathrm{S}, \mathrm{F}}$ have been verified by measurements in different houses and apartments, but additional measurements are going on to increase the number of available data sets to confirm the $M_{\mathrm{S}, \mathrm{F}}$ values in different environments.

\subsection{Comparison of the mains symmetry factor with the mode conversion factor}

In a white paper from CISPR/I/PLT (December 2006), a new method has been presented to measure of the mode conversion factor (MCF) shown in Fig. 6. A symmetrical signal is applied to the input. The load itself is the metallic box that is described in Part 1 of

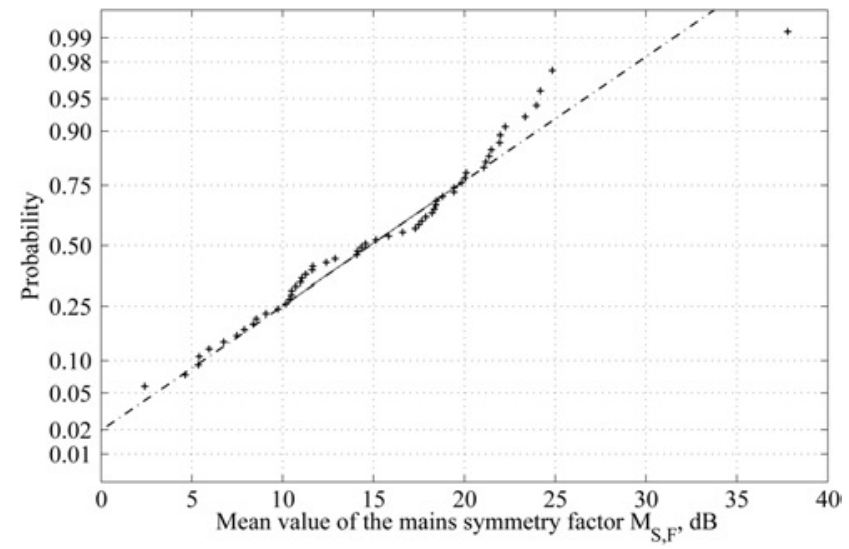

Figure 4 Normal probability plot of mean value of mains symmetry factor shows a mean value of $15 \mathrm{~dB}$ (cross-phase)

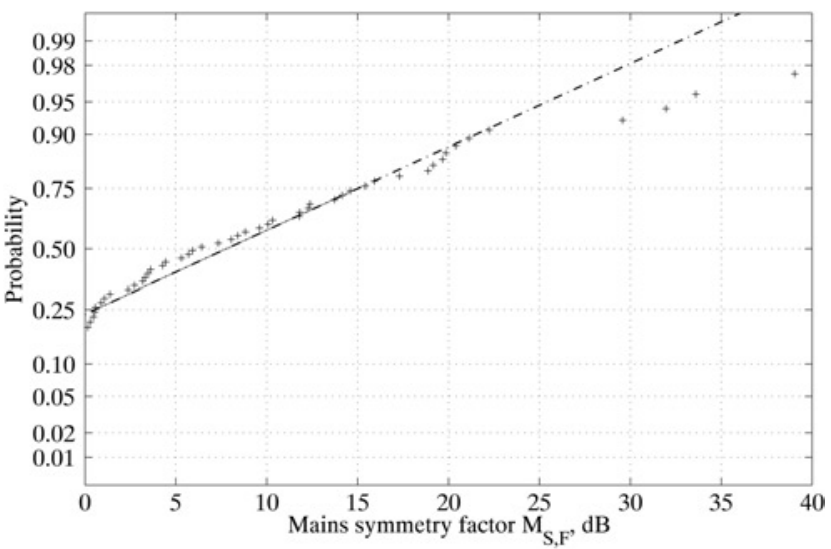

Figure 5 Normal probability plot of the symmetry decoupling factor for the socket 9 (on a single phase)

the paper. The differential and common-mode currents from the mains to the box are measured. The mode conversion factor is the ratio of both currents at the same socket and using the measuring set-up as given in Fig. 2 of Part 1. The measured results are shown in Fig. 6

$$
\mathrm{MCF}_{\text {output }, i}=I_{\text {sym }, \text { out }, i}-I_{\text {asym }, \text { out }, i}
$$

The MCF method only delivers valid results (in $\mathrm{dB}$ ) for the mains symmetry factor or the HF symmetry of a given network, if the condition $A_{\text {asym }}>A_{\text {sym }}$ (Case 1) is satisfied during the measurements. If not, the correct corresponding $M_{\mathrm{S}, \mathrm{F}}$ values could be calculated, nevertheless using the model depicted in Fig. 1b, and taking into account the attenuation of the dominating coupling path, P1 or P2. Without these corrections, the MCF method can only be regarded as a method to analyse the HF properties of a one-port network that does not deliver valid results for a typical two-port network such as a mains network or a part thereof.

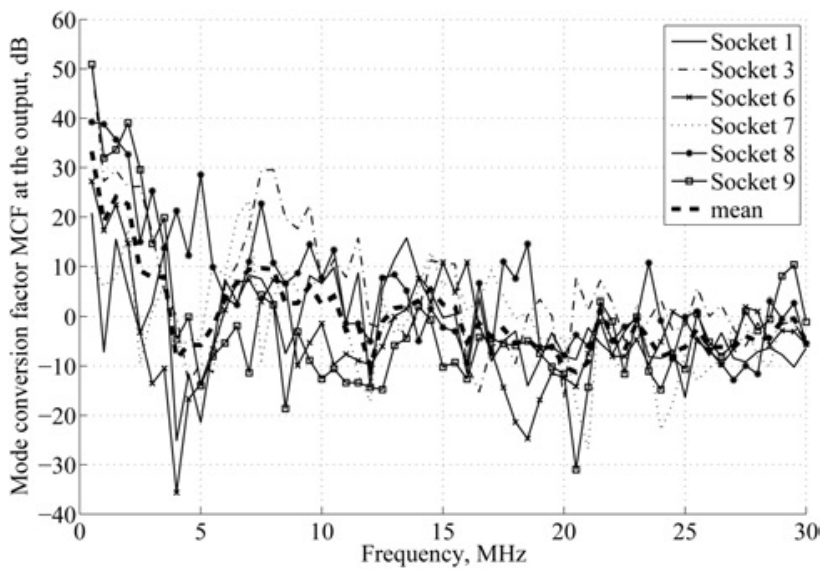

Figure 6 Mode conversion factor at the output sockets 

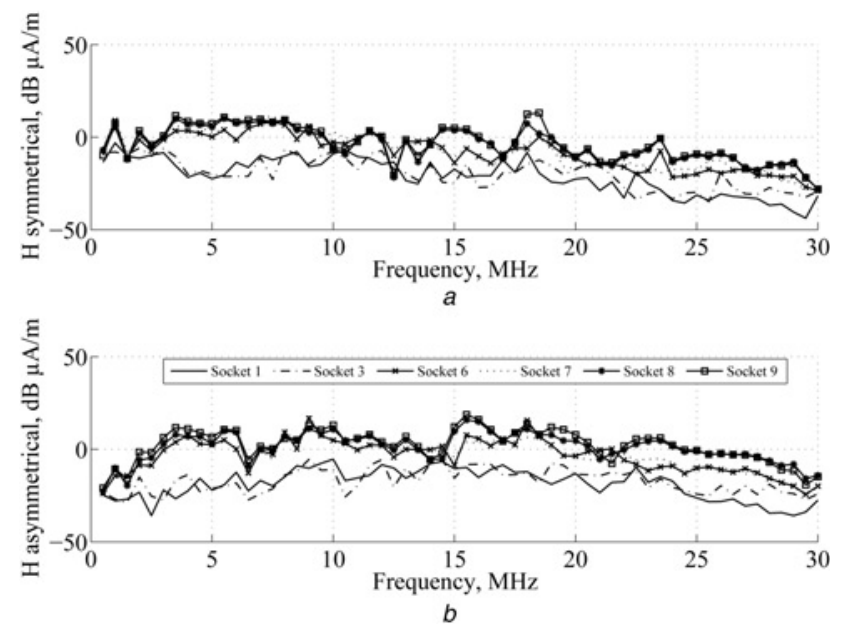

Figure 7 Magnetic field strength at the different load positions

a Symmetrical feeding

$b$ Asymmetrical feeding

\subsection{Comparison of the mains symmetry factor based on currents and magnetic field strength}

In addition to the measurement of the currents at the load, the maximum of the magnetic field strength has been measured with an active loop antenna for symmetrical and asymmetrical feeding (Fig. 7). The maxima are obtained by the geometric sum of the measurements at $3 \mathrm{~m}$ distance from the mains line in all three orthogonal orientations of the loop

$$
\begin{aligned}
\bar{M}_{\mathrm{S}, \mathrm{F}, \mathrm{M}} & =\frac{1}{n} \\
& \sum_{i=1}^{n}\left(\left(I_{\text {sym }, \text { in }}-H_{\mathrm{sym}, \mathrm{out}}\right)-\left(I_{\text {asym }, \text { in }}-H_{\text {asym }, \text { out }}\right)\right)
\end{aligned}
$$

The measurement results (in $\mathrm{dB}$ ) can be split into two groups for symmetrical and asymmetrical feeding:
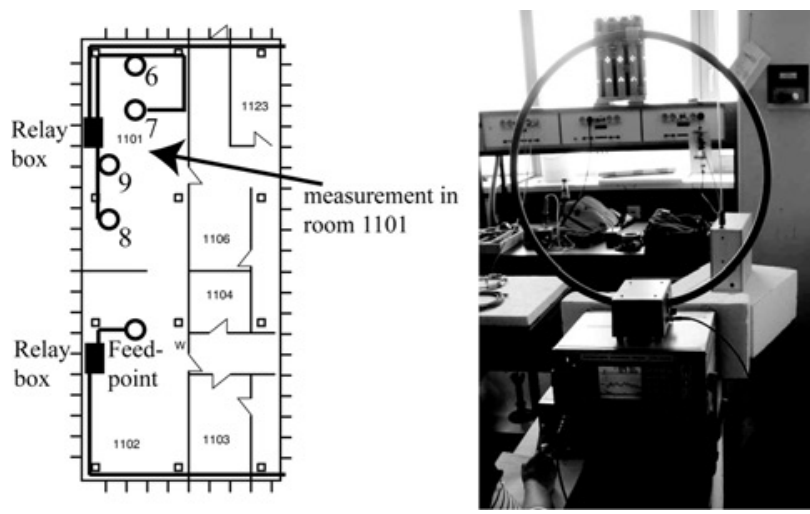

Figure 8 Measurement of the magnetic field strength in room 1101 with an active loop antenna

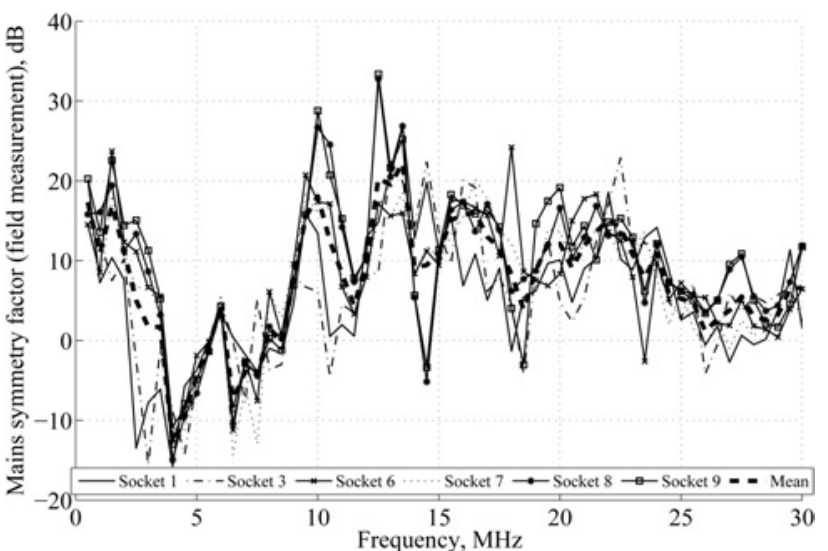

Figure 9 Mains symmetry factor measurements based on the magnetic field strength

(a) all sockets in room 1101 and (b) sockets 1 and 3. The equivalent behaviour of the measurements at sockets $6-9$ is a result of the special line configuration in room 1101. The mains network forms a part of a loop that carries the same common-mode current (Fig. 8). Therefore the measured magnetic field strength is nearly equivalent for the different sockets. The resulting $M_{\mathrm{S}, \mathrm{F}, \mathrm{H}}$ based on the magnetic field strength is depicted in Fig. 9. The correlation between the $M_{\mathrm{S}, \mathrm{F}}$ based on currents and the $M_{\mathrm{S}, \mathrm{F}, \mathrm{H}}$ based on the magnetic field strength, as shown in Fig. 10, is quite high. This connection is obvious because both quantities are related to each other by the Biot and Savart's law. The dominating cause for the magnetic field is the common-mode current flowing on the mains.

\section{Design of an applicable T-ISN}

The symmetry of the T-ISN is normally described by a longitudinal conversion loss value. This value depends on the differential- and common-mode impedances

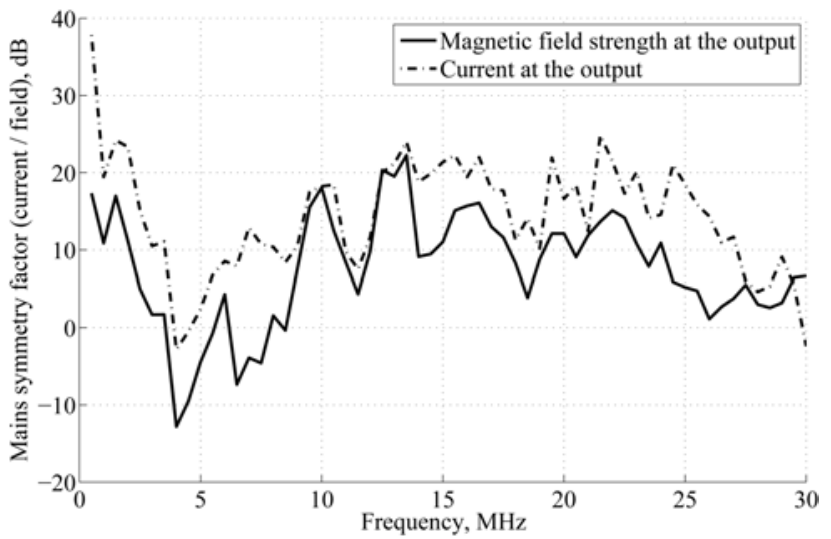

Figure 10 Comparison of the mains symmetry factor based on the current at the load or the magnetic field strength at the load 
during the calibration. The used impedances in a real installation normally differ from the equipment under test (EUT) impedances. The differential-mode rejection (DMR), however, measures the relation between the differential- and common-mode terminal voltages directly at the EUT Port of the T-ISN. The unbalance defining components of the T-ISN could be designed to simulate the measured average DMR of $\sim 15 \mathrm{~dB}$ in accordance with the findings for the mean value of mains symmetry factor $M_{\mathrm{S}, \mathrm{F}}$ of this COST action for cross-phase measurements or the smaller value for measurements on the same phase.

\section{Conclusions}

In the first part of this paper, a new measurement method has been presented to describe the attenuation and the HF symmetry characteristics of the mains network. The reason for the development of the mains decoupling factor $D_{M}$ has been explained and the necessary changes of the measurement set-up are pointed out to reproduce the disturbance scenario of present day. In this second part, the differences and similarities between the mains conversion factor and the mains symmetry factor $M_{\mathrm{S}, \mathrm{F}}$ have been illustrated and analysed. Measurements show a $M_{\mathrm{S}, \mathrm{F}}$ of the order of $15 \mathrm{~dB}$ for cross-phase measurements and $\sim 7.5 \mathrm{~dB}$ on the same phase. Further measurements are necessary to confirm these $M_{\mathrm{S}, \mathrm{F}}$ values. These measurements should apply the presented measurement method taking into account all requirements regarding the impedances. The mains symmetry factor is important to characterise the HF symmetry of the mains network and to build T-ISN for EMC compliance tests of BPL or telecommunication devices with an appropriate symmetry.

\section{Acknowledgments}

Major parts of this work are based on the results obtained from [4]. The measurements and further improvements of the measurement set-up emerged from the European Cooperation in the field of Scientific and Technical Research COST 286 with the topic 'Electromagnetic compatibility (EMC) in diffused communications systems'. The Joint Technical Action 2 (JTA2) deals with the 'EMC analysis of LF unstructured telecom networks'. Further details can be found in [5]. We are glad to recognise the help and assistance of the European Action COST 286 Team throughout the investigations under this Joint Technical Action.

\section{References}

[1] Battermann S, GaRBe h: 'Interference potential of PLC and measurement of mains characteristics'. Proc. 18th Int. Wroclaw Symp. EMC, Wroclaw, Poland, June 2006

[2] SHIOZAWA H, WATANABE Y, TOKUdA M: 'Calculation of radiated emission from the power line by 4-terminal pair network theory'. IEEE Proc. Int. Symp. EMC, August 2005, vol. 3, pp. 996-1001

[3] MIYOSHI K, KUWABARA N, AKIYAMA Y, ET AL.: 'Calculation of radiating magnetic field from indoor ac main cable using four-port network'. IEEE Proc. Int. Symp. EMC, August 2005, vol. 3, pp. 1002-1007

[4] Available at: http://www.bundesnetzagentur.de/ media/archive/6578.zip, accessed June 2007

[5] Special workshop on BPL (organized by COST 286), EMC Europe, Barcelona, Spain, 4-8 September 2006, available at: http://www.emc.york.ac.uk/cost286/, accessed June 2007 